\title{
BLOGS CIUDADANOS EN LAS ELECCIONES ESPAÑOLAS DE 2008: ASIMILACIÓN DE LA AGENDA Y REPRODUCCIÓN DE LA LÓGICA PARTIDISTA
}

\author{
Lidia Valera Ordaz \\ (Universitat de València) \\ lidia.valera@uv.es
}

\begin{abstract}
Resumen: El presente estudio se propone dilucidar las características de la blogosfera política española durante la campaña de 2008 mediante una muestra de tres reputados blogs ciudadanos y esclarecer si se produce una discusión ciudadana independiente de la agenda partidista y mediática. El análisis de contenido permitirá identificar el tipo de cobertura que estos blogs realizan sobre la campaña a través de las siguientes variables: enlaces, temas principales, protagonistas y valoraciones de los autores. Las conclusiones sugieren una significativa fragmentación de la blogosfera en espacios ideológicos, carentes de conexión entre sí y tendentes a la polarización del discurso.
\end{abstract}

Palabras clave: Campaña electoral, elecciones generales 2008, weblogs, opinión pública, NTIC.

Abstract: The present study intends to elucidate the main features of the Spanish political blogosphere through a sample of three renowned citizen blogs, as well as to clarify if an independent public discussion takes place regardless of the party and media agenda. The content analysis seeks to establish how these blogs covered the campaign with the help of the following variables: linking behavior, main topics discussed, main characters and authors' personal opinions. The results suggest a stark ideological division within the Spanish blogosphere, with no cross-ideological links between blogs and a tendency to discourse polarization.

Keywords: Electoral campaign, General Elections 2008, weblogs, public opinion, ICT.

\section{INTRODUCCIÓN}

a creación de la Web 2.0 ha propiciado el surgimiento de un nuevo espacio público digital que convive con el espacio público tradicional, más estructuralmente jerarquizado y organizado en torno a la preponderancia de los medios de comunicación de masas como principales intermediarios entre una ciudadanía crecientemente despolitizada (Putnam, 2000) y una clase política que goza de niveles muy bajos de aceptación ciudadana.

La política no ha permanecido al margen de esta revolución digital. Los partidos y candidatos políticos se han lanzado a generar contenido en la Red. De este modo, los nuevos instrumentos de la Web 2.0 se han convertido en armas primordiales de 
Blogs ciudadanos en las elecciones españolas de 2008: asimilación de la agenda y reproducción de la lógica partidista

marketing político, a través de las cuales las formaciones partidistas tratan de seducir a su electorado, ofreciendo una imagen de cercanía y transparencia.

Las redes sociales, los portales de vídeo, los foros, los blogs y, por supuesto, los sitios web de los partidos políticos están ampliando las fronteras de la comunicación política tradicional, históricamente canalizada a través de los medios de comunicación a una audiencia masiva y, ocasionalmente, en espacios de comunicación interactivos "cara a cara" limitados por definición a un número reducido de interlocutores (Maarek, 2009: 176), en su mayoría militantes.

La diversificación de la oferta comunicativa y el libre acceso a la producción de contenidos online son políticamente relevantes en tanto que permiten a un inusitado volumen de usuarios hacerse oír en el espacio virtual. En este sentido, este nuevo espacio digital se perfila para algunos como la esperanza regeneradora de la anquilosada dinámica institucional y mediática que obstaculiza la participación ciudadana en un debate libre entre iguales (Jenkins, 2008).

La presente investigación se enmarca en un proyecto de $1+D^{1}$ más amplio que ha realizado un estudio en profundidad sobre la campaña cibernética del 2008. Mi contribución a este proyecto radica en un análisis pormenorizado de tres destacados weblogs ciudadanos, con el fin de estudiar sus características formales básicas, el tratamiento de la campaña que realizan, los temas y enfoques que priorizan en sus publicaciones, las valoraciones que realizan sobre los actores políticos, así como la orientación ideológica que se deriva de los elementos anteriores.

A través de un enfoque cuantitativo, se realiza un análisis de tres blogs ciudadanos y su seguimiento de la campaña electoral. El análisis de contenido contribuirá, por lo tanto, a resolver las dudas sobre si existe un verdadero debate político ciudadano en el espacio virtual ajeno a la dinámica de partidos o sí, por el contrario, tal como sugieren los resultados de esta investigación, la blogosfera política española reproduce hasta la fecha las coordenadas del discurso partidista y polarizado que preside el espacio público offline.

\section{BLOGS COMO HERRAMIENTAS DE COMUNICACIÓN POLÍTICA}

La revolución provocada por la Web 2.0 y la facilitación de los procesos de edición de contenidos digitales han abierto la puerta a nuevas herramientas comunicativas, que han permitido a personas anónimas convertirse en lo que Gillin llama 'los nuevos influyentes': individuos que consiguen una presencia señalada en el ciberespacio público gracias a las nuevas tecnologías (2009: 13). De hecho, muchos bloggers constituyen hoy en día líderes de opinión del espacio virtual

\footnotetext{
${ }^{1}$ Ciberdemocracia en la campaña electoral de 2008 en España. El uso ciudadano de las nuevas tecnologías para la percepción, deliberación y movilización electorales", dirigido por el catedrático de Periodismo de la Universidad Rey Juan Carlos Víctor Sampedro Blanco. Proyecto financiado por el Ministerio de Educación y Ciencia (2008-2010).
} 
capaces de concitar nutridos seguidores.

De este modo, los blogs han adquirido protagonismo, en tanto en cuanto proporcionan interesantes marcos interpretativos especializados para los periodistas, una fuente de información cotidiana y una fecunda cantera de temas (Drezner y Farrell, 2007).

Los medios digitales operan con principios diferentes al modelo de los mass media característico de la Modernidad: acceso, colaboración, reciprocidad o comunicación entre iguales son algunos de los estándares de la red. En este sentido, la blogosfera norteamericana ha actuado en más de una ocasión como perro guardián de los medios de comunicación, denunciando falsas noticias: en 2004, sin ir más lejos, diversos blogs conservadores pusieron en jaque a la cadena CBS, que en su programa 60 minutes había acusado al presidente Bush de eludir sus responsabilidades en su paso por el ejército mediante influencias familiares, una acusación de la que la cadena tuvo que disculparse después de que varios bloggers demostraran que era totalmente infundada.

En el escenario español, los blogs irrumpieron en el espacio público a raíz de los dramáticos atentados del 11-M y los días previos a las elecciones del 14-M, caracterizados por una alta demanda informativa y una situación de desconcierto y desconfianza hacia la información facilitada por el gobierno. El afán por conocer la autoría del atentado llevó a muchos individuos a indagar en la red, de forma que el consumo de cibermedios, foros de debate y blogs experimentó un crecimiento geométrico (Sampedro y López, 2005: 126-143).

\subsection{Blogs en campaña}

Algunos autores sugieren que en la blogosfera encuentra expresión el descontento de parte de la ciudadanía frente a los actores políticos, las rutinas periodísticas y el sistema de funcionamiento democrático (Jenkins, 2008: 217-219) y que este nuevo espacio público virtual, en el que hay cabida para el diálogo respetuoso y recíprocamente ilustrado, es susceptible de desencadenar un empoderamiento político entre individuos previamente despolitizados. Sin embargo, los estudios revelan una tendencia ligeramente antagónica: son los individuos previamente ideologizados y políticamente activos los que tienden a ser más activos en el espacio virtual (Davis, 2001: 19).

Con todo, "Internet puede facilitar la entrada en la política activa a ciudadanos anteriormente inactivos políticamente. Sin embargo, las motivaciones y el entorno social que radicalizan a los individuos son, en gran parte, independientes de la tecnología que puedan acabar empleando para acceder a la militancia política" (Seoane y Jones, 2008: 239).

En cualquier caso, el blog ha sido una herramienta tempranamente importada al ámbito de la política por sus amplias posibilidades comunicativas, las nulas barreras de entrada y su potencial para alcanzar a una gran cantidad de usua- 
Blogs ciudadanos en las elecciones españolas de 2008: asimilación de la agenda y reproducción de la lógica partidista

rios. Los blogs saltan a la arena política con motivo de la innovadora campaña de Howard Dean en 2004, que reúne abundante financiación mediante un uso estratégico de Internet y convierte su modesta campaña en un trampolín hacia la nominación. A través de su blog, Dean envía por primera vez en una contienda electoral mensajes constantes a sus seguidores, desplegando una exitosa estrategia de contacto permanente con sus bases. Tal como recuerda Jenkins, "Dean recaudó más dinero en la red a base de pequeñas contribuciones que ningún candidato anterior (...). Su equipo utilizaba los blogs para crear una relación más íntima en tiempo real con sus seguidores "(2008: 212).

A partir de 2004, el uso de blogs se incorpora a la dinámica de campaña estadounidense, y además se integra en la prensa digital como un elemento indiscutible de comunicación con los ciudadanos. Esta tendencia se extiende a otros países europeos, como atestigua el éxito de la candidata socialista Segolène Royal en las primarias francesas de 2007 con su apuesta cibernética Désirs d'avenir, que le sirvió de gran ayuda para consolidar su candidatura en las presidenciales (Maarek, 2009: 298).

En España, la campaña de 2008 experimenta la generalización del blog como instrumento de marketing político: un $27 \%$ de los cabezas de lista provinciales de los dos principales partidos contaron con uno al inicio de la campaña (Peytibi, Rodríguez y Gutiérrez-Rubí, 2008: 28). Además, surgieron los llamados agregadores de blogs, plataformas de lectura de las bitácoras ideológicamente afines que eran accesibles desde el propio sitio web del partido, y que creaban además un sentimiento de comunidad entre bloggers (Red Liberal y Red Progresista son las principales).

Con el creciente protagonismo de los blogs en el espacio público, diversos estudios han puesto de manifiesto que los blogs se constituyen como compartimentos estancos, ideológicamente homogéneos, sin ningún contacto con ideologías contrapuestas. En una investigación que recrea el mapa de la blogosfera política estadounidense de 2004, Adamic y Glance descubrieron que los blogs liberales y conservadores no tenían prácticamente ningún contacto entre ellos y que sólo enlazaban con sus homólogos políticamente semejantes (2005). Otros han señalado que los enlaces cruzados entre blogs de opciones políticas diversas adoptan en la mayoría de los casos la forma del desdén y el desprecio hacia el oponente (Hargittai, Gallo y Kane, 2008).

Una investigación ${ }^{2}$ sobre la blogosfera política francesa en colaboración con el periódico Le Monde ha cartografiado la distribución de sus enlaces desde 2007 hasta 2011 en función de su orientación ideológica y ha arrojado los mismos

\footnotetext{
${ }^{2}$ En este texto, referencio el artículo de Guilhem Fouetillou que comenta la investigación, mientras que en la bibliografía aparece también referenciada la investigación completa: Fouetillou, Guilhem (2011). De 2007 à 2011, l'évolution de la blogosphère politique, Le Monde, 4/7/2011 (http://politicosphere.blog. lemonde.fr/2011/07/04/de-2007-a-2011-levolution-de-la-blogosphere-politique/) (19/09/2011).
} 
resultados: los blogs se aglutinan en zonas segmentadas e ideológicamente homogéneas, que corresponden a los distintos partidos políticos y apenas presentan enlaces con grupos ajenos (Foulleitou, 2011).

En el caso español, el estudio de López y Lara confirma que gran parte de estas bitácoras devienen espacios para el discurso radical y polarizado, cajas de resonancias ideológicas en las que se dan cita soflamas partidistas. "El análisis cualitativo ha puesto de manifiesto que, con escasas excepciones, los blogs de éxito en el campo de la política tienden a competir con sus mentores ideológicos en su radicalismo. Muchos blogs funcionan como mera herramienta de reverberación de los líderes de opinión políticos y mediáticos" (López y Lara, 2009: 49).

\section{MUESTRA DE BLOGS}

El estudio que presento se centra en el análisis de los tres blogs ciudadanos que aparecen recogidos a continuación: a) K-Government: blog de análisis político que ha evolucionado hacia un portal web dedicado a reflexionar sobre las redes sociales y la democracia participativa; b) Contando Estrelas: blog de un ciudadano gallego (Elentir) que combina análisis políticos y campañas de agitación con artículos de crítica cultural; c) A sueldo de Moscú: Ex concejal de Izquierda Unida en Rivas-Vaciamadrid y en la actualidad director de comunicación del mismo ayuntamiento. Progresista y militante de IU. La vinculación explícita del autor a IU, como veremos más adelante, afecta decisivamente al enfoque que realiza sobre la campaña electoral.

Tabla 1. Muestra de blogs.

\begin{tabular}{|l|l|c|}
\hline Nombre del blog & \multicolumn{1}{|c|}{ Autor } & Fecha de creación \\
\hline Contando Estrelas & Elentir & $15 / 07 / 2004$ \\
\hline $\begin{array}{l}\text { A Sueldo de } \\
\text { Moscú }\end{array}$ & $\begin{array}{l}\text { Ricardo J. Ro- } \\
\text { yo-Villanova }\end{array}$ & $01 / 12 / 2004$ \\
\hline K-Government & Carlos Guadián & $08 / 10 / 2003$ \\
\hline
\end{tabular}

Fuente: Elaboración propia.

La selección de este corpus de investigación se realizó a través de dos criterios fundamentales:

1. Antigüedad. Los ciudadanos anónimos carecen de una relevancia pública capaz de granjearles una rápida popularidad en la Red (a diferencia de los actores políticos y de los medios de comunicación), por lo que requieren de una cierta trayectoria y una constancia de publicación para alcanzar cierta visibilidad y consolidarse en la blogosfera. En la muestra seleccionada, dos bitácoras fueron creadas en el año 2004 (Contando Estrelas y A Sueldo de Moscú) y la otra en el 2003 (K-Government), de 
Blogs ciudadanos en las elecciones españolas de 2008: asimilación de la agenda y reproducción de la lógica partidista

forma que las tres presentan un dilatado recorrido que acredita su buen posicionamiento en la blogosfera política española para el marco temporal estudiado.

2. Buen posicionamiento en Technorati. Este buscador y base de datos de weblogs (el más completo de Internet) es, además, una útil herramienta de estimación de la incidencia de un blog, que funciona por medio del algoritmo Authority calculando la importancia relativa de cada bitácora sobre todo en base a los enlaces que recibe y a la procedencia y relevancia de éstos (el criterio es ascendente: a mayor número de enlaces, mayor autoridad). El hecho de que un blog esté muy enlazado implica que, además de ser leído en abundancia, sus publicaciones están bien consideradas por el resto de bloggers e internautas (Maratea, 2008: 143).

Los tres blogs seleccionados, tal como se refleja en la tabla 5, poseen un formidable posicionamiento en el marco temporal estudiado, especialmente meritorio tratándose de autores anónimos que han ido adquiriendo su estatus paulatinamente: Contando Estrelas y A Sueldo de Moscú presentan cifras por encima de los 1000 enlaces entrantes (valores en torno a cien ya reflejan una relevancia moderada en la blogosfera), lo que los convierte en figuras consolidadas del panorama digital con un acervo destacado de lectores y seguidores, muy por delante de K-Government, que concita 634 enlaces.

Ambos criterios avalan la centralidad de estas tres bitácoras en la blogosfera política española, justificando el interés científico de analizar el enfoque que estos blogs ciudadanos realizan de la campaña electoral.

\section{METODOLOGÍA}

\subsection{Marco temporal}

El análisis que presento se limita a un marco temporal que transcurre desde el 1 de enero hasta el 10 de marzo de 2008. Además, el análisis se subdivide en dos arcos temporales sucesivos: del 1 de enero hasta el 21 de febrero de 2008 (precampaña), y del 22 de febrero hasta el 10 de marzo (campaña oficial, jornada de reflexión, día de los comicios y jornada posterior).

\subsection{Metodología de análisis}

En cuanto al a metodología de análisis, el punto de partida de este estudio es el método clásico de análisis de contenido (Krippendorf, 1990), si bien éste ha sido adaptado a las particularidades de la comunicación digital y enriquecido con variables que recogen la especificidad del blog como herramienta de comunicación, y que se refieren, entre otros, a los enlaces, el blogroll o el uso de multimedia (Díaz Noci y Palacios, 2007).

Asimismo, conviene señalar que el diseño metodológico utilizado en este es- 
tudio, que a continuación se detalla, fue concebido y empleado en el marco del citado Proyecto de Ciberdemocracia para el análisis de un corpus mucho más amplio de bitácoras (López García et al., 2011).

El texto que presento a continuación es el producto de un esfuerzo analítico, centrado en la codificación de las diferentes variables de análisis en una base de datos configurada con Microsoft Access.

Las variables desarrolladas al efecto del análisis propuesto son las siguientes:

1. Características generales: formato y extensión de las entradas, fecha de publicación, uso de multimedia, número de artículos publicados y ritmo de publicación.

2. Enlaces: presencia de blogroll o listado permanente de hipervínculos, número de enlaces salientes, ideología de éstos, y número de enlaces entrantes.

3. Comentarios: se contabilizó el promedio diario y por post de comentarios en cada uno de los blogs durante los dos arcos temporales sucesivos: precampaña y campaña.

4. Artículos: todos los contenidos publicados en estos tres blogs durante el marco temporal señalado han sido codificados de acuerdo con tres subvariables:

Asimismo, se analizan los siguientes aspectos:

1. Temas: 54 temas de actualidad fueron definidos para englobar el conjunto de cuestiones relevantes que se trataron en la campaña. Esta lista incluía asuntos políticos de fondo (como la política exterior, el desempleo o la política fiscal), cuestiones relativas al propio devenir de la campaña (debates electorales, sondeos, estimación de resultados) y temas de estricta actualidad. En la codificación se registraron desde uno hasta un total de tres temas diferentes por artículo.

2. Protagonistas: partidos políticos, candidatos, cargos públicos y otros actores sociales engrosaban una lista de 49 protagonistas posibles de los artículos analizados. Se codificaron nuevamente entre uno y tres protagonistas por artículo.

3. Valoración de los protagonistas: se evaluaba la valoración que los autores hacían de los distintos protagonistas de sus posts por medio de tres categorías: positiva, negativa y neutral, sin atender a gradaciones ni intensidades con el fin de simplificar la estimación de la orientación ideológica de los responsables de los blogs. 
Blogs ciudadanos en las elecciones españolas de 2008: asimilación de la agenda y reproducción de la lógica partidista

\section{RESULTADOS}

\subsection{Características generales}

La actualización y la constancia son condiciones indispensables para obtener presencia en la red, concitar una comunidad de lectores habituales y de este modo conseguir cierta incidencia en el espacio público virtual. Aunque los blogs no tienen una periodicidad rígida a la que ajustarse, como sucede en los medios de comunicación de masas, la disparidad entre frecuencias de publicación en los casos estudiados resulta necesariamente llamativa, tal como refleja la tabla 2 , que registra el volumen total de posts publicados en los dos tramos del marco temporal y su ritmo diario de publicación.

Tabla 2. Artículos publicados durante precampaña y campaña. Ratio diario.

\begin{tabular}{|l|l|l|}
\hline \multicolumn{1}{|c|}{ Blogs } & \multicolumn{1}{|c|}{$\begin{array}{c}\mathbf{N}^{\circ} \text { posts precampaña } \\
\text { (ratio diario) }\end{array}$} & \multicolumn{1}{|c|}{$\begin{array}{c}\mathbf{N}^{\circ} \text { posts campaña } \\
\text { (ratio diario) }\end{array}$} \\
\hline Contando Estrelas & $254(4,88)$ & $89(5,23)$ \\
\hline A sueldo de Moscú & $(1,48)$ & $24(1,41)$ \\
\hline K-Government & $38(0,73)$ & $9(0,53)$ \\
\hline
\end{tabular}

Fuente. Elaboración propia.

Las diferencias de actividad de los tres blogs son bastante acusadas. Contando Estrelas lidera el ranking de forma contundente con un promedio diario de publicación de 4,88 posts, que se ve aumentado en la etapa propiamente dicha de campaña a un 5,23 de artículos al día. Estamos, por lo tanto, ante un usuario muy prolífico que manifiesta un alto grado de compromiso con la creación y actualización de su blog.

Los otros dos ofrecen un ritmo de publicación mucho más modesto. A Sueldo de Moscú presenta una tasa de actividad superior a la periodicidad diaria (que en cualquier caso no resiste comparación con la fecundidad de Contando Estrelas), mientras que K-Government no alcanza esta frecuencia y se sitúa por debajo del artículo diario. Además, frente al aumento de actividad del blog de Elentir durante la campaña oficial, las bitácoras de Carlos Guadián y Ricardo Royo-Villanova experimentan un ligero declive en el caso del segundo y más importante en el primero.

El hecho de que dos de estos blogs ciudadanos presenten una tasa de actividad por encima del artículo diario constata un distanciamiento significativo ( $y$ palmario en el caso de Contando Estrelas) de los tradicionales criterios de periodicidad de las publicaciones impresas, en concreto, de la lógica del columnismo periodístico (un artículo al día o a la semana) que, sin embargo, continúa gobernando la publicación de contenidos de reputados blogs de periodistas españoles (López García y Clemente, 2010). 
No obstante, la fuerte discrepancia en actividad se compensa levemente con la disparidad de extensión de los artículos. Los blogs no están constreñidos espacialmente como ocurre en la prensa, con lo que parten de una libertad mucho mayor a la hora de elaborar su contenido. Frente al $28 \%$ de posts de más de una pantalla de extensión de Contando Estrelas, el $49 \%$ de contenidos de A Sueldo de Moscú y el $77 \%$ de K-Government poseen una extensión semejante. En el caso del blog gallego y en lógica respuesta a su extraordinaria fecundidad, el grueso de las publicaciones son más limitadas y se circunscriben a una pantalla $(65 \%)$.

En cuanto a la multimedialidad de los contenidos, Contando Estrelas destaca por ser el que mayor diversidad de formatos ofrece a sus lectores: un $68 \%$ de sus artículos contienen imágenes, principalmente fotografías, y un $28 \%$ incluyen clips de vídeo. Los otros dos blogs se muestran más tímidos en esta materia, si bien K-Government muestra una especial atención a los gráficos e infografías para complementar sus artículos.

\subsection{Enlaces.}

Los enlaces constituyen la quintaesencia de la comunicación digital, en tanto que son responsables de la hipertextualidad. De hecho, los blogs nacieron precisamente como listados de enlaces de recomendaciones entre internautas. Analizar el blogroll de cada bitácora nos permite, por lo tanto, descubrir los principales intereses y la orientación ideológica de su autor, atendiendo al tipo de preferencias de lectura que éste proporciona a sus lectores de forma permanente en el listado de hipervínculos de su diario digital. En el caso que nos ocupa, merece la pena subrayar la gran cantidad de hipervínculos que integran el blogroll de K-Government (212), que duplica al número de los otros dos, tal como se plasma en la tabla 3.

Tabla 3. Composición del blogroll, tipos de sitios web enlazados.

\begin{tabular}{|l|c|c|c|c|}
\hline \multicolumn{1}{|c|}{ Blogs } & $\begin{array}{c}\text { Oros } \\
\text { blogs }\end{array}$ & Medios & Otros & Total \\
\hline Contando Estrelas & 52 & 6 & 29 & 87 \\
\hline A Sueldo de Moscú & 67 & 3 & 12 & 82 \\
\hline K-Government & 189 & 11 & 12 & 212 \\
\hline
\end{tabular}

Fuente. Elaboración propia.

La tabla 4, por su parte, registra el número de enlaces de cada blogroll atendiendo a la afinidad ideológica que éstos presentan respecto a la postura ideológica del autor, definida principalmente en función del agregador de blogs en el que el autor se ha integrado voluntariamente (bien en la Red Liberal, que aglutina a los segmentos conservadores de derecha de la blogosfera española, 
Blogs ciudadanos en las elecciones españolas de 2008: asimilación de la agenda y reproducción de la lógica partidista

bien en la Red Progresista, que se ubica a la izquierda del espectro ideológico). La operación consiste en suponer que si el autor consiente en integrar su bitácora en una de estas populares redes ideológicas de la blogosfera política española es porque concurre, cuanto menos, un acuerdo ideológico de mínimos. En el caso de A Sueldo de Moscú, la condición de militante de su autor en Izquierda Unida es suficientemente ilustrativa en cuanto a sus preferencias políticas.

Tabla 4. Blogroll, enlaces según ideología.

\begin{tabular}{|l|c|c|c|c|}
\hline \multicolumn{1}{|c|}{ Blogs } & Afines & $\begin{array}{c}\text { Neutros/Otros } \\
\text { temas }\end{array}$ & Contrarios & Total \\
\hline Contando Estrelas & 63 & 24 & 0 & 87 \\
\hline A Sueldo de Moscú & 68 & 10 & 4 & 82 \\
\hline K-Government & 54 & 158 & 0 & 212 \\
\hline
\end{tabular}

Fuente. Elaboración propia.

Pues bien, en referencia a la tesis de polarización de la blogosfera a la que se ha hecho referencia con anterioridad (Adamic y Glance, 2005), esta investigación suscribe modestamente los resultados obtenidos en otros estudios. Los tres blogs que integran la muestra enlazan mayoritariamente y de forma arrolladora con sitios web ideológicamente afines, dos de ellos directamente carecen de cualquier nexo con sitios web de ideología contraria y uno, A sueldo de Moscú, constituye una humilde excepción con cuatro enlaces que conducen a páginas cuya orientación política difiere de la del autor y que, en cualquier caso, no suponen un contrapeso suficiente frente a los 68 enlaces de contenido afín. A esto se añaden hipervínculos neutros o relativos a otros temas, que destacan especialmente en el caso de K-Government, blog que como ya hemos visto, no sólo se ocupa de la actualidad política.

Los resultados que ha arrojado el análisis refrendan ampliamente la hipótesis de que los blogs se configuran como nodos de redes ideológicamente homogéneas y aisladas del resto del espectro político, en la línea de otros estudios, que han aportado evidencia empírica no sólo de la fragmentación ideológica de la blogosfera, sino también del hecho de que el contacto cruzado entre opciones políticas enfrentadas, cuando se produce, no siempre obedece a los estándares de cordialidad y educación exigidos por el debate público, sino que con frecuencia adopta un tono de explícito desdén (Hargittai, Gallo y Kane, 2008).

Por otra parte, conviene observar quién enlaza a cada uno de ellos para dilucidar la notoriedad y el grado de centralidad que ocupa un weblog en el ecosistema digital. Para esta última tarea he recurrido al buscador de blogs Technorati, por considerarlo la herramienta más fiable de medición de la influencia de un blog gracias a su algoritmo Authority, que calcula la importancia relativa de cada bitácora en base a los enlaces que recibe y a la procedencia de éstos. 
Tabla 5. Enlaces entrantes, según Technorati.

\begin{tabular}{|l|c|}
\hline \multicolumn{1}{|c|}{ Blogs } & Enlaces entrantes \\
\hline Contando Estrelas & 1165 \\
\hline A Sueldo de Moscú & 1205 \\
\hline K-Government & 634 \\
\hline
\end{tabular}

Fuente. Elaboración propia.

Los datos, que ya hemos anticipado, muestran que estamos ante tres blogs con una muy significativa incidencia sobre el espacio público digital. Contando Estrelas y A Sueldo de Moscú presentan una influencia muy semejante, de forma que casi duplican la notoriedad de K-Government. Ambos ocupan un lugar nuclear en sus respectivas esferas ideológicas: Contando Estrelas, en la de la derecha conservadora y A Sueldo de Moscú, en el ámbito de la izquierda más progresista. Estamos, por lo tanto, ante ciudadanos que se han convertido en líderes de opinión del ciberespacio, y que como tales cuentan con sólidas comunidades de incondicionales.

\subsection{Comentarios}

Otro criterio para indagar la notoriedad de un blog consiste en prestar atención al número de comentarios que genera entre los lectores. En este estudio, se registró el volumen total de comentarios obtenidos por cada blog a lo largo de los dos tramos sucesivos del marco temporal, y se calculó el ratio por post para cada uno de ellos, tal como queda recogido en la tabla 6.

Tabla 6. Comentarios durante precampaña y campaña, ratio diario y ratio por post.

\begin{tabular}{|l|c|c|c|c|}
\hline \multicolumn{1}{|c|}{ Blogs } & $\begin{array}{c}\mathbf{N}^{\circ} \text { Comentarios } \\
\text { precampaña y } \\
\text { ratio diario }\end{array}$ & $\begin{array}{c}\text { Comentarios } \\
\text { precampaña, } \\
\text { ratio por post }\end{array}$ & $\begin{array}{c}\mathbf{N}^{\circ} \text { comentarios } \\
\text { campaña y } \\
\text { ratio diario }\end{array}$ & $\begin{array}{c}\text { Comentarios } \\
\text { campaña, ra- } \\
\text { tio por post }\end{array}$ \\
\hline $\begin{array}{l}\text { Contando } \\
\text { Estrelas }\end{array}$ & $2668(51,3)$ & 10 & $1200(66,66)$ & 13,48 \\
\hline $\begin{array}{l}\text { K-Gover- } \\
\text { nment }\end{array}$ & $109(2,09)$ & 3,02 & $37(2,05)$ & 4,11 \\
\hline $\begin{array}{l}\text { A Sueldo } \\
\text { de Moscú }\end{array}$ & $1893(36,40)$ & 34,41 & $733(40,72)$ & 20,36 \\
\hline
\end{tabular}

Fuente. Elaboración propia. 
Blogs ciudadanos en las elecciones españolas de 2008: asimilación de la agenda y reproducción de la lógica partidista

En primer lugar, llama la atención el enorme flujo de comentarios que concitan Contando Estrelas y A Sueldo de Moscú con 51,3 y 36, 4 comentarios al día, lo que no deja de constituir un éxito para dos ciudadanos anónimos y contribuye a acreditar la mentada centralidad de ambas bitácoras en sus esferas ideológicas respectivas. Especialmente llamativo es el número de comentarios por post del blog de Ricardo Royo-Villanova durante la precampaña, que asciende a 34,41 comentarios y revela, de nuevo, la existencia de una nutrida comunidad de seguidores capaz de generar esa media de comentarios por cada entrada publicada. Por su parte, K-Government obtiene un número de comentarios mucho más modesto. Una posible explicación de esta marcada discrepancia, más allá de la diversa centralidad de cada bitácora según Technorati, podrían ser las desigualdades en "intensidad ideológica" de cada blog. Tal como evidencia el análisis temático a continuación, frente a la mayor moderación y distancia de K-Government respecto a la agenda temática de su esfera ideológica, Contando Estrelas y A Sueldo de Moscú presentan posicionamientos ideológicos mucho más fervorosos y comprometidos, con lo que serían más capaces de movilizar a sus respectivas redes ideológicas.

Se observa una tendencia alcista a medida que se aproximan los comicios, observable en el aumento de los ratios diarios entre los dos marcos temporales (con la salvedad de K-Government que se mantiene estable), que se explica fundamentalmente en base al mayor grado de interés y participación que tienden a experimentar los lectores ante la proximidad de las elecciones, puesto que en situaciones caracterizadas por una importante intensidad emocional, como es una campaña, el público tiende a participar más activamente (Sampedro y López, 2005: 145-152).

\subsection{Artículos}

Cabe hacer una matización inicial con respecto al corpus: las tres bitácoras prestan abundante atención a las elecciones, pero a lo largo del marco temporal de la codificación, no sólo se abordan asuntos relacionados con la campaña electoral, sino también temas ajenos a los comicios. Con el fin de acotar el análisis, se ha optado por aplicar una variable previa a todos los artículos: contenido relacionado con la campaña y, por lo tanto, por codificar únicamente los artículos relacionados. De este modo, el corpus asciende a 171 artículos: 14 correspondientes a K-Government, 61 procedentes de A Sueldo de Moscú y 96 de Contando Estrelas.

\subsubsection{Temas}

Este análisis parte de 54 variables de temas relativos a la campaña, si bien a continuación algunas categorías aparecen agrupadas: "Economía" integra "Impuestos", "Infraestructura" y "Empleo", mientras que "Campaña" aglutina "Estra- 
tegias", "Eventos de campaña" y "Organización", si estas variables no aparecen reflejadas individualmente.

Gráfico 1. Dispersión temática en Contando Estrelas.

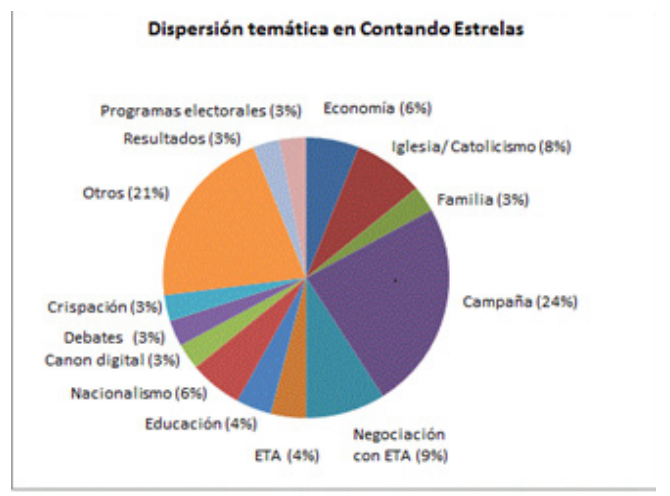

Fuente. Elaboración propia.

En Contando Estrelas, los asuntos relativos al desarrollo de la campaña electoral ocupan una cuarta parte de la cobertura temática (24\%). Además, el blog se hace eco de uno de los hitos más notorios de la campaña 2008: los debates electorales entre los candidatos a la presidencia (3\%).

Asimismo, Contando Estrelas se ocupa de forma notoria de asuntos que habían dominado la agenda política de la derecha principalmente en el primer binomio de la legislatura 2004-2008: como son la "Familia" (3\%) y la "Iglesia / Catolicismo" (8\%). Los tres habían sido temas esgrimidos con saña y encono por parte del PP en su crítica contra la legalización del matrimonio homosexual y su defensa de la familia tradicional y del matrimonio entendido como institución natural y sagrada entre el hombre y la mujer. Elentir se suma plenamente a esta postura, otorgándole un protagonismo inusitado en un periodo en el que estos asuntos ya habían sido significativamente desplazados de la agenda popular y mediática.

Destaca, asimismo, la atención concedida al terrorismo de ETA (4\%), que no obstante se explica parcialmente por la irrupción del asesinato del ex concejal del PSE Isaías Carrasco en plena campaña. Sin embargo, la importancia dedicada a la polémica negociación que el Gobierno de Zapatero mantuvo con ETA (9\%) a lo largo de la legislatura resulta muy llamativa. El empeño del Gobierno socialista por enmarcar esta negociación como un "proceso de paz" y el atentado de la banda terrorista en diciembre de 2006 en la terminal 4 del aeropuerto de Barajas poniendo fin al "alto al fuego permanente" se tradujo en una crítica implacable y sostenida por parte de la oposición conservadora que acusó al Ejecutivo de realizar concesiones políticas a la banda y de traicionar a las víctimas del terrorismo. Ambos temas, junto con la cobertura del nacionalismo (6\%), revelan que, 
Blogs ciudadanos en las elecciones españolas de 2008: asimilación de la agenda y reproducción de la lógica partidista

en lo que a la cobertura temática de la campaña se refiere, se ha producido una asimilación parcial de la agenda política de la derecha.

Gráfico 2. Dispersión temática en K-Government.

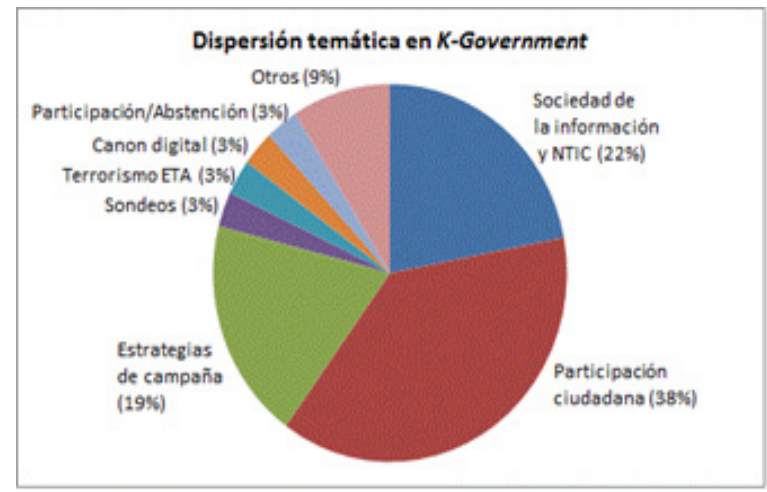

Fuente. Elaboración propia.

En el caso de K-Government, las estrategias partidistas concitan un $19 \%$ de la cobertura temática, junto con un $3 \%$ dedicado a los clásicos sondeos electorales, y un $3 \%$ destinado al canon digital, uno de los asuntos que más polémica generó entre los internautas en la primera legislatura de Zapatero. Sin embargo, en este caso destacan principalmente dos temas que se relacionan de forma complementaria: la participación ciudadana (38\%) y la sociedad de la información y las nuevas tecnologías (22\%). Esto sucede principalmente porque su autor, Carlos Guadián es un miembro muy activo de la blogosfera española y un entusiasta de las posibilidades de participación que ofrece Internet, con lo que realiza un minucioso seguimiento de la cibercampaña y sus novedades.

Gráfico 3. Dispersión temática en A Sueldo de Moscú.

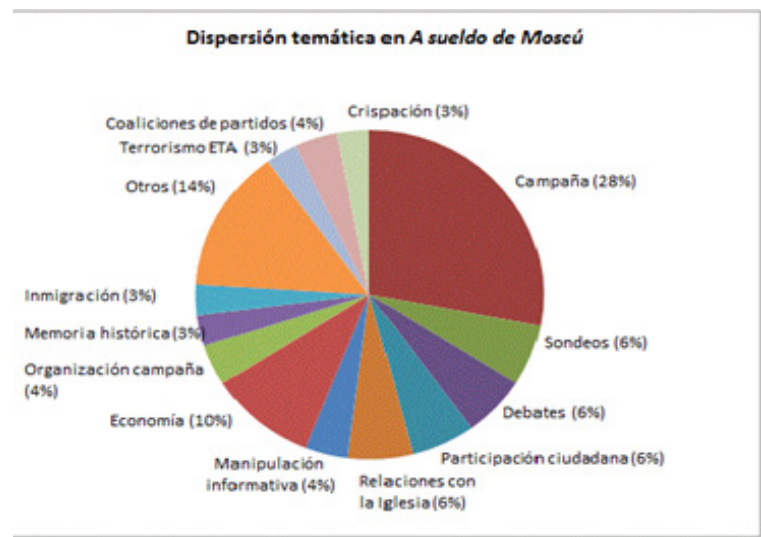

Fuente. Elaboración propia. 
Estamos ante el blog que desde el punto de vista del análisis temático menos se ve influido por la agenda política de su respectiva esfera ideológica y que más independencia mantiene en cuanto al contenido que ofrece en comparación con el resto del corpus. Con todo, el análisis y la valoración de los protagonistas confirmarán los datos anticipados por el análisis de los enlaces sobre su posicionamiento ideológico.

A sueldo de Moscú es el blog que mayor diversidad temática presenta. Dedica un volumen considerable de contenido a la campaña $(28 \%)$ y a su organización (4\%), a lo que se añade una nutrida cobertura de los sondeos (6\%) y de los debates electorales (6\%). Se observa una correspondencia significativa entre la agenda de este blog y la de la izquierda representada por IU. Destaca, sobre todo, la atención concedida a las relaciones entre Iglesia y Estado (6\%), concretamente, la reivindicación permanente de una separación rotunda entre ambos, una de las clásicas e históricas aspiraciones de la izquierda española.

En segundo lugar, el blog de Ricardo Royo-Villanova se hace eco de la debatida "Memoria histórica" (3\%), otro asunto reivindicado permanentemente por IU a lo largo de la andadura democrática y que cobra actualidad por la aprobación de la nueva ley en 2007.

\subsubsection{Protagonistas y valoración}

Este análisis parte de una lista de 49 posibles protagonistas de los artículos analizados, compuesta principalmente por candidatos, partidos políticos, y otros actores e instituciones sociales relevantes.

Gráfico 4. Protagonistas en Contando Estrelas.

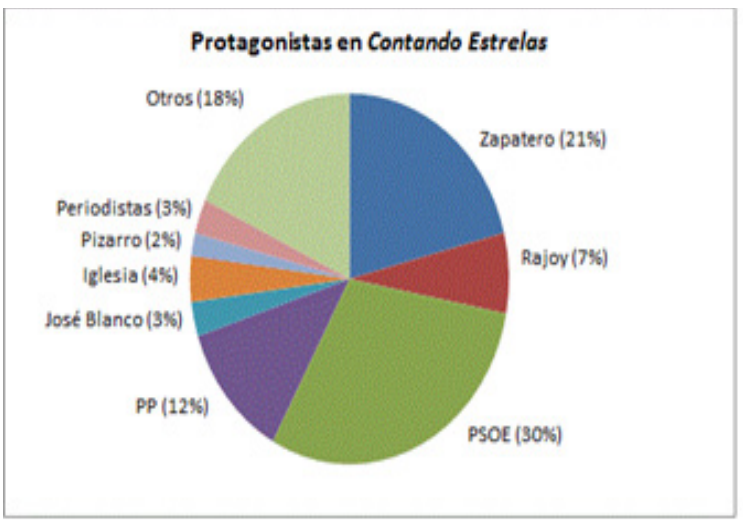

Fuente. Elaboración propia.

En Contando Estrelas, los dos partidos nacionales (42\%) y sus dos candidatos a la presidencia (28\%) protagonizan el $70 \%$ de los artículos, mientras que otras fuerzas están directamente ausentes, lo que apunta a la tendencia hacia la 
Blogs ciudadanos en las elecciones españolas de 2008: asimilación de la agenda y reproducción de la lógica partidista

consolidación del bipartidismo que experimenta el sistema de partidos español.

Llama la atención, sin embargo, que, tratándose de un blog cuyo autor se ubica ideológicamente en la esfera de la derecha, el PSOE y Zapatero obtengan una presencia muy por encima de su rival político. Dos razones lo explican. La primera remite a la creciente dinámica polarizadora del juego político en España, que desvirtúa la práctica política convirtiéndola en un ataque permanente del contrario (González y Bouza, 2009) y la segunda, a una de las tendencias más destacadas de la comunicación política actual: la creciente negativización de las campañas (Ansolabehere e lyengar, 1995).

Tabla 7. Valoración de los protagonistas en Contando Estrelas.

\begin{tabular}{|l|c|c|c|}
\hline $\begin{array}{l}\text { Valoración I } \\
\text { Protagonista }\end{array}$ & Positiva & Neutral & Negativa \\
\hline Zapatero & & 3 & 39 \\
\hline Rajoy & 13 & 3 & \\
\hline PSOE & & 3 & 58 \\
\hline PP & 21 & 4 & \\
\hline Iglesia & 5 & 2 & \\
\hline José Blanco & & & 6 \\
\hline
\end{tabular}

Fuente. Elaboración propia.

La mayor cobertura del PSOE obedece sencillamente al interés de Elentir por criticar al Gobierno de Zapatero, tal como confirma el análisis de las valoraciones de los protagonistas que se recoge en la tabla 7. No hay ni una valoración positiva del PSOE o cualquiera de sus miembros, del mismo modo que todas las evaluaciones que se realizan del PP y de su candidato a la presidencia son positivas o neutrales.

Gráfico 5. Protagonistas en K-Government.

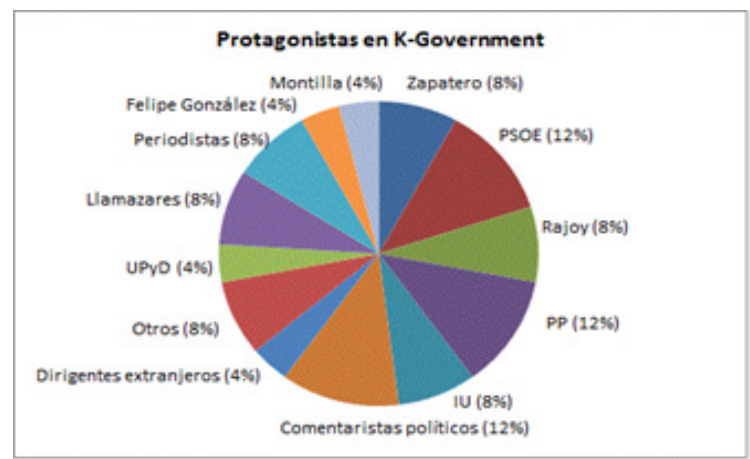

Fuente. Elaboración propia. 
Tanto es así, que la crítica de Elentir al presidente del gobierno se traduce en 39 valoraciones negativas y 58 evaluaciones del mismo signo del PSOE.

En K-Government, por otra parte, existe mayor dispersión de protagonistas. Las fuerzas minoritarias IU y UPyD obtienen una presencia significativa, un $8 \%$ y un $4 \%$ respectivamente, en comparación con PP y PSOE, y a estos protagonistas se añaden otros como comentaristas políticos, periodistas y dirigentes extranjeros. Destaca, no obstante, el protagonismo más acusado del partido socialista (Montilla, Felipe González, Zapatero y PSOE concitan un $28 \%$ ), que delata la postura ideológica del autor y que es positivamente valorado de forma global (tabla 8).

Tabla 7. Valoración de los protagonistas en Contando Estrelas.

\begin{tabular}{|l|c|c|c|}
\hline $\begin{array}{c}\text { Valoración/ } \\
\text { Protagonista }\end{array}$ & Positiva & Neutral & Negativa \\
\hline Zapatero & 2 & & \\
\hline PSOE & 2 & 1 & \\
\hline PP & & 1 & 2 \\
\hline Rajoy & & 1 & 1 \\
\hline IU & & 2 & 1 \\
\hline Llamazares & & 1 & 1 \\
\hline UPyD & & & \\
\hline $\begin{array}{l}\text { Otros miembros } \\
\text { del PSOE }\end{array}$ & 2 & & \\
\hline
\end{tabular}

Fuente. Elaboración propia.

Por su parte, A Sueldo de Moscú muestra una arrolladora sobrerrepresentación de IU y de su candidato, que conforman una presencia del $43 \%$, frente al moderado $24 \%$ del PSOE y Zapatero y el $23 \%$ del PP y Rajoy. Los tres partidos y sus candidatos protagonizan más del $80 \%$ del contenido ofrecido por Ricardo Royo-Villanova, que, como militante de IU, aprovecha su bitácora para tratar de otorgar a su partido el espacio que normalmente los medios convencionales le niegan frente a PP y PSOE. 
Blogs ciudadanos en las elecciones españolas de 2008: asimilación de la agenda y reproducción de la lógica partidista

Gráfico 5. Protagonistas en K-Government.

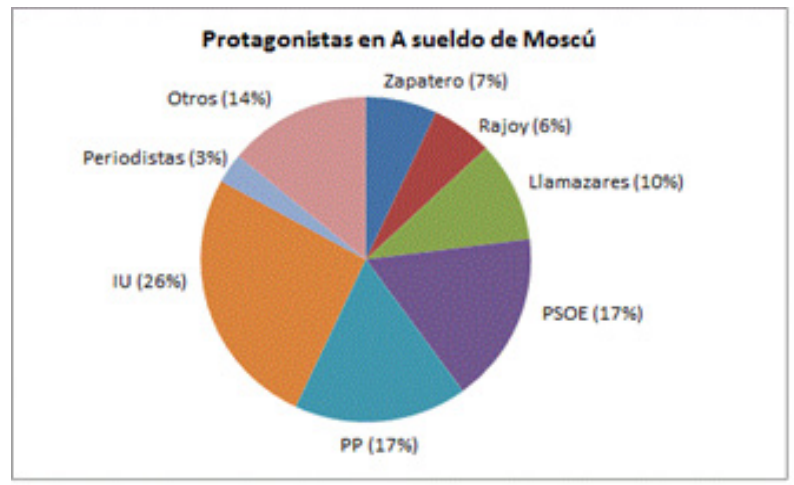

Fuente. Elaboración propia.

El partidismo explícito e intencional de A Sueldo de Moscú y su enfoque ideológico se perciben de forma cristalina en las valoraciones de los protagonistas de sus publicaciones (tabla 9). Frente a la evaluación indistintamente negativa de la derecha y de la Iglesia, destaca la postura crítica con el PSOE, al que se tiende a valorar negativamente pero se le reconoce algún mérito (con 16 evaluaciones negativas, 3 positivas y 1 neutral), y la entronización de Izquierda Unida y su candidato Gaspar Llamazares, con 28 y 11 valoraciones positivas respectivamente.

Tabla 9. Valoración de los protagonistas en A Sueldo de Moscú.

\begin{tabular}{|l|c|c|c|}
\hline $\begin{array}{c}\text { Valoración/ } \\
\text { Protagonista }\end{array}$ & Positiva & Neutral & Negativa \\
\hline Zapatero & 1 & 2 & 6 \\
\hline Rajoy & & 2 & 6 \\
\hline Llamazares & 11 & 1 & \\
\hline PSOE & 3 & 1 & 16 \\
\hline PP & & & 21 \\
\hline IU & 28 & 3 & 2 \\
\hline Iglesia & & & 1 \\
\hline
\end{tabular}

Fuente. Elaboración propia.

\section{DISCUSIÓN}

Los resultados de esta investigación cuestionan la idea de que se esté produciendo una deliberación ciudadana y democrática 2.0 en Internet independiente de la creciente polarización que preside el juego político entre los dos principales partidos nacionales del sistema político español (González y Bouza, 2009: 
129-131). Más bien sugieren que existe una asociación entre agendas y que los blogs ciudadanos tienden a reproducir la agenda marcada por sus partidos de referencia y amplificada por los medios de comunicación ideológicamente afines.

En un sistema mediático de pluralismo polarizado (Hallin y Mancini, 2008) o de bipolarización antagónica (Sampedro y Seoane, 2009), caracterizado por enormes dosis de partidismo y claramente alineado en torno al eje izquierda-derecha, no necesariamente sorprende que la blogosfera política reproduzca las características del sistema de medios español y su histórica predisposición para ser cooptado por los intereses partidistas.

De acuerdo con las conclusiones de polarización y fragmentación de la blogosfera de varias investigaciones (Sunstein, 2001; Adamic y Glance, 2005; Hargittai et al., 2008), el análisis de contenido ha puesto de manifiesto que la confrontación política se traslada también en el caso español al complejo entramado de las bitácoras políticas dentro de los límites que impone el tamaño reducido de la muestra seleccionada. Y que además lo hace mediante la reproducción parcial de la agenda temática partidista. A ello contribuye, asimismo, el uso estratégico e interesado que los partidos han hecho hasta ahora de las herramientas Web 2.0, que lejos de tratar de fomentar la deliberación democrática mediante la interacción con los usuarios, tienden a crear espacios ideológicamente homogéneos para reafirmar y difundir sus mensajes persuasivos (Sunstein, 2007: 80) (Sampedro y Seoane, 2009: 134) (Dader, 2009: 54).

Esta reproducción de la agenda política-mediática en los blogs ciudadanos resulta más evidente cuando se constata el notorio alejamiento que padecen las bitácoras que componen el corpus de los temas que más intensamente preocupan a la ciudadanía, lejos de lo que podría esperarse en primera instancia, al tratarse, al fin y al cabo, de publicaciones que en principio nada tienen que ver con las estructuras partidistas (con la salvedad de la militancia de A Sueldo de Moscú en Izquierda Unida). Según las encuestas del CIS de octubre de 2007, los asuntos más relevantes que inquietaban a los ciudadanos eran el empleo $(37,4 \%)$, la vivienda $(34,8 \%)$ y la inmigración $(32,5 \%)$. Sin embargo, en nuestro corpus, el empleo concita una atención por debajo del $3 \%$ en dos de los blogs, la vivienda está decididamente ausente en todos ellos y la inmigración sólo aparece recogida en un $3 \%$ en A sueldo de Moscú, mientras que sí aparecen profusamente recogidos varios temas de las respectivas agendas políticas de la derecha y la izquierda: la negociación del Gobierno con ETA, las relaciones Iglesia-Estado, la familia y el nacionalismo.

Este alejamiento de los blogs que son objeto del estudio de los temas que más intensamente preocupan a la ciudadanía discurre paralelo al patente divorcio entre la agenda ciudadana y la mediática, que aparece reflejado en un informe de Francesc Pujol sobre la cobertura de la campaña electoral en 20 medios (Pujol, 2008). González y Bouza llegan a la misma conclusión en su estudio sobre las elecciones de 2008, que compara la agenda mediática con las preocupaciones ciudadanas que emergen a través de varios grupos de discusión. El desfase entre 
Blogs ciudadanos en las elecciones españolas de 2008: asimilación de la agenda y reproducción de la lógica partidista

ambas agendas es clamoroso: el sobredimensionamiento de cuestiones como el terrorismo en los medios contrasta con poderosas inquietudes ciudadanas, como la inmigración, que apenas aparecen recogidas en los medios ni en los programas electorales (González y Bouza: 2009).

En definitiva, los resultados indican que se ha producido la traslación de la dinámica polarizadora del juego político a la blogosfera junto con las dosis de negativismo que prevalecen en la comunicación política moderna (Ansolabehere e lyengar, 1995). De hecho, el blog Contando Estrelas es concebido por su autor como una plataforma de ataque permanente al rival político, dedicando gran parte de su contenido a este propósito, tal como ha puesto de manifiesto el análisis de contenido, en términos de cobertura mediática y valoraciones de los actores políticos. A Sueldo de Moscú, por su parte, constituye un clásico ejemplo de bitácora que nace con el clarísimo objetivo de defender el ideario de un partido político que en los medios convencionales no encuentra tanto predicamento por la creciente dinámica bipartidista.

En cualquier caso, el análisis de protagonistas y valoraciones evidencia el clarísimo posicionamiento ideológico de cada bitácora, que lleva a una evaluación positiva de la ideología propia y a una crítica implacable de la ajena, confirmando las tesis de Sunstein sobre fragmentación y polarización ideológica en la red (2001: 59-69). Lo que en última instancia invita a sospechar que la participación ciudadana en la blogosfera española, lejos de constituir una deliberación pública o un diálogo ciudadano enriquecido respecto del espacio mediático tradicional, ha adoptado la forma de un vehemente alineamiento ideológico no exento de exaltación y fanatismo.

\section{CONCLUSIONES}

La nueva esfera pública, generada al albor del impacto de las nuevas tecnologías digitales en el sistema comunicativo, ha permitido a ciudadanos anónimos erigirse en voces influyentes del ciberespacio y cosechar una influencia moderada sobre la opinión pública, tal como ha puesto de manifiesto el análisis de los enlaces entrantes y de los comentarios de los blogs que conforman la muestra de este estudio, capaces en algunos casos de alcanzar a un público que si bien no resiste comparación con el de los medios convencionales, sí resulta significativo en el entorno de la comunicación digital.

A nivel formal, se observa que dos de estos diarios digitales presentan tasas de actividad notablemente por encima del artículo diario, lo que pone de manifiesto un alejamiento de los estándares de periodicidad de los medios de masas (concretamente del columnismo impreso) y una lógica de producción mucho más ágil y dinámica.

Pese a la limitación del presente corpus, que sólo engloba a tres bitácoras, resulta imprescindible destacar que tanto el análisis de enlaces como el de la agenda temática y el de las valoraciones de los protagonistas han puesto de 
manifiesto una notable homogeneidad ideológica. Los blogs del presente estudio enlazan abrumadoramente con sitios web de ideología similar, realizan una cobertura de la campaña que prioriza los temas preferidos por los partidos que, más o menos explícitamente, apoyan; y valoran a los actores políticos de forma que determinadas candidaturas son sistemáticamente criticadas y otras obtienen permanentemente una valoración positiva. Todo ello contribuye a validar modestamente las tesis de fragmentación y polarización ideológica sobre la blogosfera de diversas investigaciones (Sunstein, 2001; Adamic y Glance, 2005; Hargittai et al., 2008; Fouetillou, 2011).

Se observa, asimismo, una tendencia al negativismo en cuanto a la evaluación de protagonistas, especialmente palmaria en el caso de Contando estrelas, por la cual los autores de los blogs se inclinan más a atacar y desacreditar al rival ideológico que a ensalzar las virtudes de sus partidos o candidatos de preferencia.

Todo ello, y a falta de estudios en profundidad y mayor innovación metodológica en este ámbito emergente de investigación, invita a reconsiderar la idea de que se esté produciendo una discusión ciudadana en Internet independiente de la dinámica de polarización que preside el juego político en España, y sugiere que la blogosfera política española reproduce la lógica del pluralismo polarizado y partidista del sistema mediático español.

\section{REFERENCIAS BIBLIOGRÁFICAS}

<http://mhcj.es/2010/12/05/guillermolopez_carolinaclemente/>

<http://www.argitalpenak.ehu.es/p291content/es/contenidos/libro/se_indice_ciencinfo/es_ ciencinf/adjuntos/ciberperiodismo.pdf>

ADAMIC, L. \& GLANCE, N. (2005): The Political Blogosphere and the 2004 U.S. Election: Divided They Blog [Consulta: 19 de Septiembre de 2011]. <http://www.blogpulse.com/papers/2005/AdamicGlanceBlogWWW.pdf>

ANSOLABEHERE, S. \& IYENGAR, S. (1997): Going Negative. How Political Advertisements Shrink \& Polarize the Electorate. New York: The Free Press.

DADER, J.L.: "Ciberpolítica en los websites de partidos políticos: la experiencia de las elecciones de 2008 en España ante las tendencias transnacionales". Revista de Sociología e Política (2009), n 34, pp. 45-62.

DAVIS, R.: "Tecnologías de la comunicación y democracia: EI Factor Internet". CIC: Cuadernos de Comunicación e Información (2001), nº 6, pp. 9-32.

DÍAZ NOCI, J. \& PALACIOS, MARCOS (2007): Ciberperiodismo: métodos de investigación. Una aproximación multidisciplinar en perspectiva comparada. [Consulta: 19 de Septiembre de 2011].

DREZNER, D. \& FARRELL, H.: "The Power and Politics of Blogs". Public Choice (2008), n' 134 , pp. 15-30.

FOUETILLOU, G. (2011): La cartographie de la blogosphère politique. Le Monde [en 
Blogs ciudadanos en las elecciones españolas de 2008: asimilación de la agenda y reproducción de la lógica partidista

línea]. 4 de julio de 2011 [Consulta: 19 de Septiembre de 2011]. <http://www.lemonde. fr/election-presidentielle-2012/visuel/2011/07/04/la-cartographie-de-la-blogosphere-politique_1544714_1471069.html>.

GILLIN, P. (2009): Los nuevos influyentes. Madrid: LID.

GONZÁLEZ, J.J \& BOUZA, F.: Las elecciones de 2008. En GONZÁLEZ, J.J \& BOUZA, F. (2009): Las razones del voto en la España democrática. Madrid: Los Libros de la Catarata, pp. 167-193.

GONZÁLEZ, J.J \& BOUZA, F.: Las elecciones de la polarización. En GONZÁLEZ, J.J \& BOUZA, F. (2009): Las razones del voto en la España democrática. Madrid: Los Libros de la Catarata, pp. 129-167.

HALLIN, D. \& MANCINI, P. (2008): Sistemas mediáticos comparados: tres modelos de relación entre los medios de comunicación y la política. Barcelona: Hacer.

HARGITTAI, E., GALLO, J. \& YALE KANE, M.: "Cross-Ideological Discussions among Conservative and Liberal Bloggers". Public Choice (2008), n 134, pp. 67-86.

JENKINS, H. (2008): Convergence Culture: La cultura de la convergencia en los medios de comunicación. Barcelona: Paidós.

KRIPPENDORF, K. (1990): Metodología de análisis de contenido: teoría y práctica. Barcelona: Paidós.

LÓPEZ GARCÍA, G. \& CLEMENTE, C. (2010): Posicionamientos ideológicos, agenda mediática y blogosfera: un análisis de la blogosfera española en las últimas elecciones generales. Miguel Hernández Communication Journal [en línea]. 5 de diciembre de 2010 [Consulta: 19 de Septiembre de 2011].

LÓPEZ, GARCÍA, G.; LARA, T. \& SÁNCHEZ DUARTE, J.M.: Los weblogs como mecanismos de reafirmación ideológica: la blogosfera española ante las Elecciones Generales de 2008. En SAMPEDRO, V. (coord.) (2011): Cibercampaña. Cauces y diques para la participación. Las elecciones generales de 2008 y su proyección tecnopolítica. Madrid: Editorial Complutense, pp. 201-236.

MAAREK, P. (2009): Marketing político y comunicación. Barcelona: Paidós.

MARATEA, R.: "The e-Rise and Fall of Social Problems: The Blogosphere as a Public Arena". Social Problems (2008), Vol. 55, n 1, pp. 139-160.

PEYTIBI, X.; RODRÍGUEZ, J.A. \& GUTIÉRREZ-RUBÍ, A.: "La experiencia de las elecciones generales del 2008”. Revista de Internet, Derecho y Política (2008), n 7, pp. 26-37.

PUJOL, F. (2008): "Informe sobre la cobertura mediática de las elecciones al Parlamento español del 9 de marzo de 2008". Politics and Media Value Project. Navarra: Universidad de Navarra. [Consulta: 19 de Septiembre de 2011]. <http://www.unav.es/econm/politics/ elecciones-9-M>

SAMPEDRO, V. \& LÓPEZ GARCÍA, G.: Deliberación celérica desde la periferia. En SAMPEDRO, V. (ed.) (2005): 13-M: Multitudes Online. Madrid: Los Libros de la Catarata, pp. 119-158.

SAMPEDRO, V. y SEOANE, F.: "Las elecciones generales españolas de 2008: 'bipolarización antagónica' fomentada por intereses político-mediáticos y las nuevas tecnologías". 
Revista de Sociología y Política (2009), n 34, pp. 129-135.

SUNSTEIN, C. (2007): Republic.com 2.0. Princeton (UK): Princeton University Press.

- (2001). República.com: Internet, democracia y libertad. Barcelona: Paidós.

\section{Breve semblanza de la autora}

Lidia Valera Ordaz es Licenciada en Periodismo (2009) y Becaria de Investigación en el Departamento de Teoría de los Lenguajes de la Universidad de Valencia desde 2010. Sus principales líneas de investigación son comunicación política, opinión pública y campañas electorales.

(Recibido el 03-10-2011; aceptado el 28-12-2011) 